\section{Self-Administration of A Headache Questionnaire in the Waiting Room Is Well Accepted By Patients and Useful For the Headache Specialist}

\section{Abstract}

Background: The diagnosis of headache rely mostly upon patient's history, but second level headache centres of the Italian NHS have to face the difficulty of a shorter availability of time, therefore we elaborated a self-administered questionnaire to be filled-in in the waiting room.

Methods: One-hundred and twenty questionnaires were studied, regarding 90 females and 30 males; mean age 41 years \pm 14 .7, mostly suffering from primary and episodic headaches.

Results: Response rates to the questionnaire were of $77.5 \%$. Responders were significantly younger than those who did not fill in the questionnaire $(39.5 \pm$ 14.3 versus $46.2 \pm 15.4, p<0.05$ ) and there were significantly more primary than secondary headache diagnoses ( $81 \%$ versus $50 \% p<0.001$ ).

Conclusion: This self-administered tool was well accepted by the patients, it was not intended to be a screening or a diagnostic tool, simply it allowed the patients to better focus on informative items of their headache, and hopefully help the Clinician to reduce visit time.

Keywords: Headache history; Headache tool; Visit time

\section{Teresa Catarci}

Azienda Sanitaria Locale Roma1 and 2,

Outpatient Headache Clinic, Rome, Italy

Corresponding author:

Teresa Catarci, MD, PhD

” teresa.catarci@aslroma1.it

Azienda Sanitaria Locale Roma1 and 2, Outpatient Headache Clinic, Rome, Italy.

Tel: +390677307581

Citation: Catarci T (2018) SelfAdministration of A Headache Questionnaire in the Waiting Room Is Well Accepted By Patients and Useful For the Headache Specialist. Int J Anesth Pain Med. Vol.4 No.1:1

Received: November 17, 2017; Accepted: November 30, 2017; Published: January 10,2018

\section{Introduction}

Headache history is of paramount importance for the correct management of headache disorders, hence a detailed and time consuming collection of the patient's medical history is required in the clinical setting [1]. Second level headache centres of the Italian National Health Service often face the difficulty of a short availability of time per patient, where little time remains for physical examination and counselling. The collection of headache history could be facilitated by the use of headache diaries, also recommended by all the main clinical guidelines for headache [24]. They can be filled in both retrospectively (e.g. in the waiting room) and prospectively before the follow-up visit; nevertheless, the information collected represent just a fragment of the whole picture, being more useful as a follow-up instrument rather than a diagnostic tool. Equally, validated screening tests (e.g. HIT-6 [5], HURT [6], Midas [7] etc.) are mainly conceived to measure headache disability, in order to raise patients' concern about their headache and seek medical attention.

Therefore, we managed to elaborate a self-administered questionnaire to be filled in while the patients are in the waiting room, in order to gather the basic sets of information prior to the visit. Since, at the best of our knowledge, there are no similar reports in the literature, we decided to study patient's compliance and comprehension of any single item, in order to eventually reshape a final, more informative questionnaire.

\section{Materials and Methods}

The questionnaire was built using most of the ICDH-II criteria for primary headaches [8], where the items were transformed into questions both in Italian and English, due to the frequent attendance of refugee patients from Nigeria, Bangladesh and Pakistan to our outpatient's clinic. All questions were formulated 
to be easily understood and quickly filled out. The questionnaire was distributed by the acceptance nurse to all the patients who attended for the first time an outpatient headache clinic of the Italian Regional Health Service, located in the centre of Rome (Azienda Sanitaria Locale ROMA 1). It was then filled in, after giving written informed consent, during the stay in the waiting room. Since the questionnaire was not designed to be a diagnostic tool per se, all items were reviewed during the visit, checked with the patients and completed, if they were deemed scarcely or not informative, by the headache specialist (T. Catarci). All patients admitted to the clinic from October 2014 (the day of the inauguration of the headache centre) to June 2015 were included in the study, their questionnaires were collected and the following items analysed: characteristics of the patients (gender, diagnosis [8] ), adherence to the questionnaire (percentage of the questionnaires filled in totally or partially), quality of the information provided by the filled in questionnaires (percentage and type of non-informative items). We calculated statistical significance using $\mathrm{Chi}^{2}$ for comparing gender and diagnoses differences between those who filled in and those who failed to fill the questionnaires, and t test to compare age between the two groups. We used Z score to calculate statistical differences between males and females and diagnoses in the group of patients who filled in the questionnaire completely. Two-sided $p$ values less than 0.05 were considered as statistically significant.

\section{Results}

One-hundred-twenty patients were admitted to the outpatient clinic, 90 females (75\%) and 30 males (25\%), mean age 41 years \pm 14.7 (range $18-80$ years), mostly suffering from primary and episodic headaches (Table 1). Among the 106 patients with primary headache, there were 46 patients suffering from migraine without aura, 9 probable migraine and 8 migraine with aura; there were also 3 cluster headache patients and 15 with tension type headache; figures in percentage of all primary headache are shown in Figure 1.

Fourteen patients only were diagnosed as having secondary headaches: mostly $(n=5)$ with headache attributed to a substance or its withdrawal, others $(n=3)$ with headache or facial pain attributed to disorder of eyes, and $(n=3)$ with headache attributed to non-vascular intracranial disorder, one patient had headache attributed to cranial or cervical vascular disorder, one to head and/or neck trauma and one to psychiatric disorder (Fig 2).

Response rates to the questionnaire were of $77.5 \%$ (93 out of 120 patients), in fact twenty-seven patients only did not fill in the questionnaire, two of whom because of language barriers. Responders were significantly younger than those who did not fill

Table 1 Patients' characteristics ( $N=120)$.

\begin{tabular}{|c|c|c|}
\hline AGE (mean \pm SD) & \multicolumn{2}{|c|}{$41 \pm 14.7$} \\
\hline GENDER & Males 30 (25\%) & Females $90(75 \%)$ \\
\hline \multirow{2}{*}{ DIAGNOSIS } & Primary H/A 106 (88\%) & Secondary H/A 14 (12\%) \\
\cline { 2 - 3 } & Episodic 90(75\%) & Chronic 30 (25\%) \\
\hline
\end{tabular}

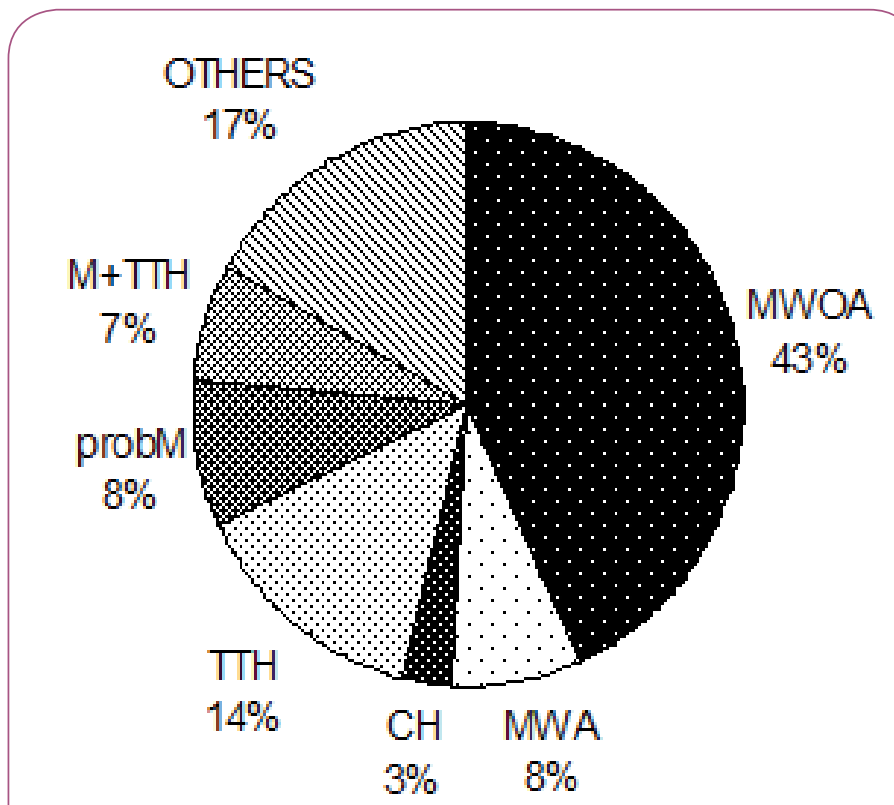

Figure 1 Type of primary headache $(n=106)$.

Table 2 Patients' acceptance of the questionnaire.

\begin{tabular}{|c|c|c|c|}
\hline & QS NOT FILLED IN & QS NOT FILLED IN & $p$ ( $t$ test) \\
\hline Patients No. & $93(77.5 \%)$ & $27(22.5 \%)$ & \\
\hline AGE \pm SD & $39.5 \pm 14.3$ & $46.2 \pm 15.4$ & $<0.05^{*}$ \\
\hline Qs: questionnaires & & & \\
\hline
\end{tabular}

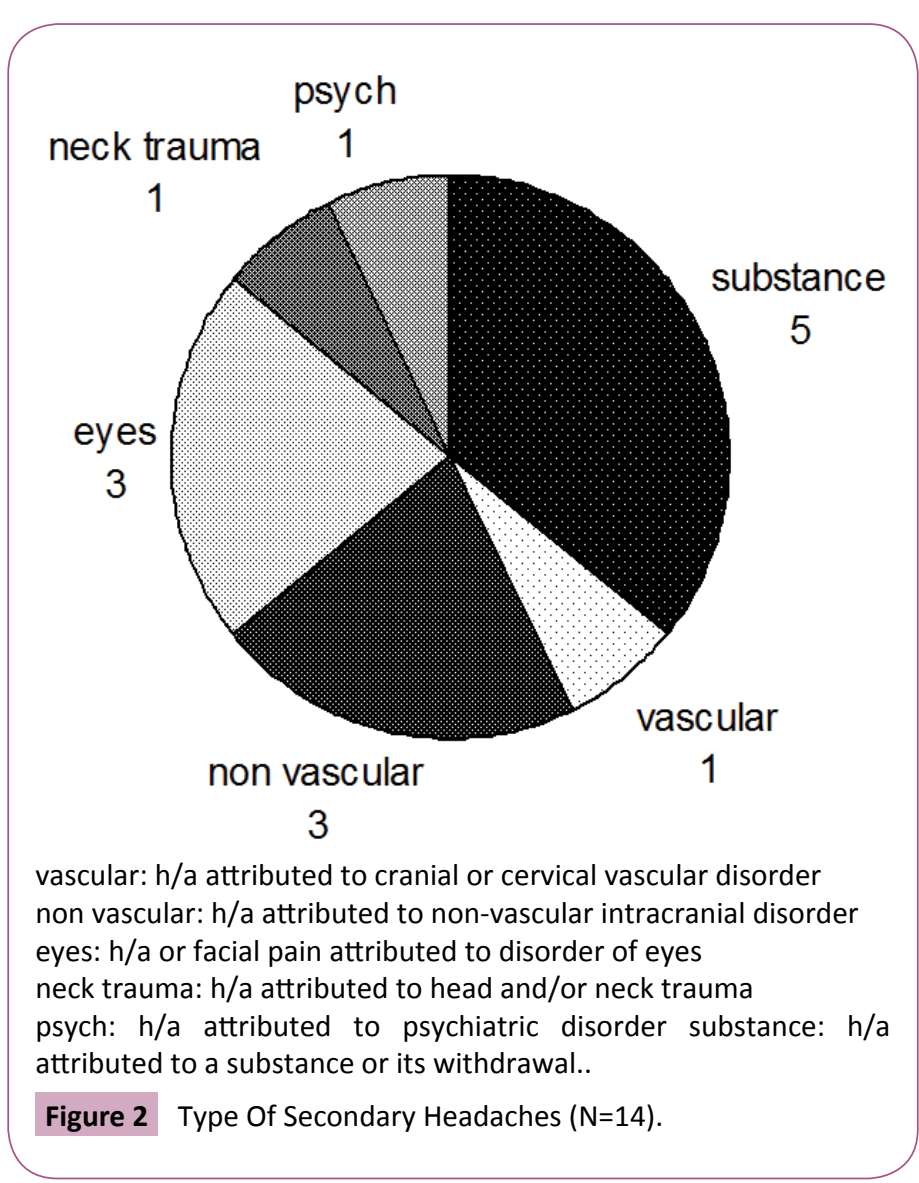


in the questionnaire $(39.5 \pm 14.3$ versus $46.2 \pm 15.4, p<0.05)$ (Table 2). The analysis of the characteristics of the 93 patients who filled in the questionnaire, showed no gender differences $(80 \%$ of the total males $(n=24 / 30)$ and $77 \%$ of the total females $(n=69 / 90)$, similar headache's trend $(80 \%$ of the episodic $(n=72 / 90)$ and $70 \%(n=21 / 30)$ of the chronic headache, although there were significantly more primary than secondary headache diagnoses $(81 \%(n=86 / 106)$ versus $50 \%(n=7 / 14) p<0.001)$ (Figure 3).

Seventeen patients only filled in completely the questionnaire (11 females and 6 males), there were no statistically significant differences between the proportion of males and females and headache diagnoses (Table 3 ). The patients were significantly younger $(34.8 \pm 14$ years versus $40.5 \pm 14, p<0.05)$ than those who filled in the questionnaire partially.

The items that were left unanswered more frequently were No. 16 ("is there anything that triggers your headache"?), 17 ("what makes your headache worse?") and 18, ("what makes your headache better?"), respectively in $40 \%, 48 \%$ and $48 \%$ of the patients. The item that failed to provide useful information for the diagnosis was No. 12 "describe what the headache is like", where non informative information were reported by $52 \%$ of the patients who described their pain as "severe", "steady", "sharp" or "constant". The best described item was No. 14 "is there any symptom that comes together with the headache like tearing of your eyes, sickness or vomit, bright light and noise bother you?" Item number 19 "What kind of painkillers do you take? Have you

Table 3 Quality of filled-in questionnaires-1: characteristics of the patients who filled-in the Qs completely.

\begin{tabular}{|c|c|c|c|}
\hline Patients No. & 17/93 (18\%) & & P (Z score) \\
\hline $\mathrm{AGE}($ mean $\pm \mathrm{SD})$ & $34.8 \pm 14$ & & \\
\hline GENDER & $\begin{array}{c}\text { Males } \\
6 / 30(20 \%)\end{array}$ & $\begin{array}{c}\text { Females } \\
11 / 90(12.2 \%)\end{array}$ & 0.29 \\
\hline \multirow{2}{*}{ DIAGNOSIS } & $\begin{array}{c}\text { Primary H/A } \\
15 / 106(14.1 \%)\end{array}$ & $\begin{array}{c}\text { Secondary H/A } \\
2 / 14(14.3 \%)\end{array}$ & 0.99 \\
\hline & $\begin{array}{c}\text { Episodic } \\
14 / 90(15.5 \%)\end{array}$ & $\begin{array}{l}\text { Chronic } \\
3 / 30(10 \%)\end{array}$ & 0.45 \\
\hline
\end{tabular}

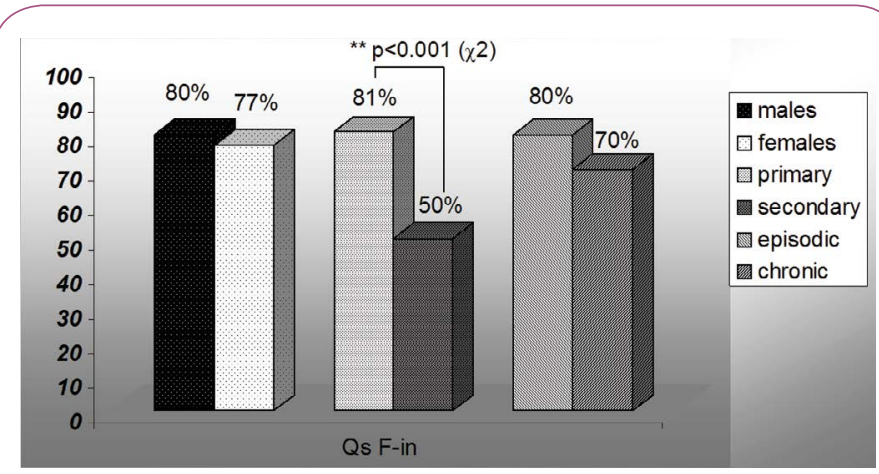

**primary headaches $81 \%$ (86 out of 106 patients); secondary headaches $50 \%$ ( 7 out of 14 patients)

Figure 3 Characteristics of the patients who filled in the Questionnaire ( $n=93)$. ever been prescribed treatments for your headache?" was left unanswered in only $9 \%$ of the questionnaires.

\section{Discussion}

The characteristics of the population studied were very much similar to those reported in other headache centres as far as age and gender distribution. In fact, recent Brazilian [9], Swiss [10] and Austrian [11] casistic reported mean ages of respectively $40.7,39.3$ and 41.1 years and female prevalence of 80,79 and $72 \%$. Our patients (mean age 41 years-old, female prevalence $72 \%)$ suffered mainly from primary $(88 \%)$ and episodic $(75 \%)$ headaches, where $66 \%$ were migraines, $14 \%$ tension type and $3 \%$ cluster headache.

Patients' acceptance of the questionnaire was very good since $77.5 \%$ of them filled in, even though only $18 \%$ did it completely. The patients who filled the questionnaire suffered mostly from primary headache probably due to the fact that, overall, it was designed to collect information more suitable to that kind of diagnosis. The small proportion of patients who filled in the questionnaire completely was much younger (34.8 \pm 14 years old) while gender and diagnoses were equally distributed.

The item that needed further investigation during the visit was "describe what the headache is like" (Item No. 12) and "what makes your headache better" (Item No. 18) since most patient wrote uninformative description like "steady" or "severe" in the first instance or left the question unanswered. Therefore, the final questionnaire was reshaped to include a more detailed question for item 12 . Other items were mostly informative, like for example item no. 19 "What kind of painkillers do you take? Have you ever been prescribed treatments for your headache?" probably because it is deemed important by the patients for his/ her headache's future management.

Overall patient's acceptance of the questionnaire was very good, although a small proportion of them filled in it completely, probably due to their shorter time spent in the waiting room, which is notably very variable. Nevertheless, this was not a limitation, since our questionnaire was not designed to be a screening tool, but rather a supplement to allow the patient to focus on the characteristics of their headache prior to the interview with the headache specialist and eventually help the latter in the management of those seen for the first time. Last, but not least, it probably allows the headache specialist to gather headache history in a less time-consuming way. In fact, even though this aspect was not investigated during the study, it happened that, soon after the end of it, it became very unpractical to stop using the questionnaire since there was a sensible increase of the time dedicated to the visit. Further studies could address this issue in the future, through a control group of patients visited without the help of the questionnaire. 


\section{References}

1 Sances G, Catarci T (2011) Management of headache patients. In: Nappi $G$ and Moskowitz M (editors) Handbook of Clinical Neurology: Headache. Amsterdam. Elsevier pp:127-135.

2 Silberstein SD, Holland S, Freitag F, Dodick DW, Argoff C, et al (2012) Evidence-based guideline update: pharmacologic treatment for episodic migraine prevention in adults: report of the Quality Standards Subcommittee of the American Academy of Neurology and the American Headache Society. Neurology 78:1337-1345.

3 Pringsheim T, Davenport W, Mackie G, Worthington I, Aubé M, et al. (2012) Canadian Headache Society guideline for migraine prophylaxis. Can J Neurol Sci 39:S1-59.

4 Steiner TJ, Paemeleire K, Jensen R, Valade D, Savi L, et al. (2007) European principles of management of common headache disorders in primary care. European Headache Federation; Lifting the Burden: The Global Campaign to Reduce the Burden of Headache Worldwide; World Health Organization. J Headache Pain 1:S3-47.

5 Yang M, Baum RR, Varon SF, Kosinski M (2010) Validation of the Headache Impact Test (HIT-6 TM) across episodic and chronic migraine. Cephalalgia 31:357-367.
6 Buse DC, Sollars CM, Steiner TJ, Jensen RH, Al Jumah MA, et al. (2012) Why HURT? A review of clinical instruments for headache management. Curr Pain Headache Rep 16:237-254.

7 Stewart WF, Lipton RB, Dowson AJ, Sawyer J (2001) Development and testing of the Migraine Disability Assessment (MIDAS) Questionnaire to assess headache-related disability. Neurology 56: 20-28.

8 Headache Classification Subcommittee of the International Headache Society (2004) The International Classification of Headache Disorders. Cephalalgia 1: 1-160.

9 Felício AC, Bichuetti DB, Santos WA, Clecio de Oliveira Godeiro Junior, Marin LF (2006) Epidemiology of primary and secondary headaches in a Brazilian tertiary-care center. Arq Neuropsiquiatr 64:41-44.

10 Kozak S, Gantenbein AR, Isler H, Merikangas KR, Angst J, et al. (2005) Nosology and treatment of primary headache in a Swiss headache clinic. J Headache Pain 6:121-127.

11 Zebenholzer K, Andree C, Lechner A, Broessner G, Lampl C, et al. (2015) Prevalence, management and burden of episodic and chronic headaches-a crossectional multicentre study in eight Austrian headache centres. Journal Headache Pain 16:46. 